\title{
Tangence
}

\section{SAID. Un portrait}

\section{Nima Mina}

Numéro 59, janvier 1999

Écrivains d'ailleurs

URI : https://id.erudit.org/iderudit/025995ar

DOI : https://doi.org/10.7202/025995ar

Aller au sommaire du numéro

Éditeur(s)

Tangence

ISSN

0226-9554 (imprimé)

1710-0305 (numérique)

Découvrir la revue

Citer cet article

Mina, N. (1999). SAID. Un portrait. Tangence, (59), 112-120.

https://doi.org/10.7202/025995ar

Ce document est protégé par la loi sur le droit d'auteur. L'utilisation des services d'Érudit (y compris la reproduction) est assujettie à sa politique d'utilisation que vous pouvez consulter en ligne.

https://apropos.erudit.org/fr/usagers/politique-dutilisation/
Cet article est diffusé et préservé par Érudit.

Érudit est un consortium interuniversitaire sans but lucratif composé de l'Université de Montréal, l'Université Laval et l'Université du Québec à Montréal. Il a pour mission la promotion et la valorisation de la recherche. https://www.erudit.org/fr/ 


\title{
SAID. Un portrait
}

\author{
Nima Mina
}

A Lorraine $V$.

\section{Note liminaire sur SAID}

Né en 1947 à Téhéran, SAID (pseudonyme) arrive en 1965 à Munich, comme étudiant. Bientôt, il s'engage dans le mouvement de protestation des étudiants iraniens qui s'est formé depuis quelques années en Allemagne. Puisqu'il est identifié comme opposant au régime du shah, son retour en Iran n'est plus possible. Un des plus éminents représentants de la littérature allophone en Allemagne, il a reçu de nombreux prix littéraires, dont le "Premio letterario internazionale Jean-Monnet", le Prix Adalbert-von-Chamisso et la Médaille Hermann-Kesten du Centre PEN allemand. Parmi ses publications les plus connues, citons: Moi et le shab. La confession de l'Ayatollab. Drames radiopboniques (Ich und der Schah. Die Beichte des Ayatollah, Hörspiele, Perspol Verlag, Hambourg, 1987); Poèmes d'amour (Liebesgedichte, P. Kirchheim-Verlag, Munich, 1989); Alors je crie jusqu'au silence (Dann schreie ich bis Stille ist, Heliopolis Verlag, Tübingen, 1990); Autoportrait pour une mère lointaine (Selbstbildnis für eine ferne Mutter, P. Kirchheim-Verlag, Munich, 1992); Où je trépasse est mon ailleurs (Wo ich sterbe ist meine Fremde, P. Kirchheim-Verlag, Munich, 1994); Le bras long des mullabs. Notes de mon exil (Der lange Arm der Mullabs. Notizen aus meinem Exil, C.H. Beck, Munich, 1995).

\section{L'engagement du poète en exil}

SAID vise d'abord l'engagement politique; il veut prévenir, informer et permettre au lecteur une attitude critique vis-à-vis de la politique. La langue de ses poèmes politiques est souvent sans aucune ornementation et se rapproche de la prose, sans toutefois garder ses distances par rapport à l'objet du discours. Il raconte et décrit des faits et des événements à partir de sa propre perspective, en renvoyant de façon concrète à son pays d'origine. 
Puisque son public est constitué de lecteurs de langue allemande, il leur parle d'une autre réalité et d'une histoire qui n'est pas la leur; par conséquent, il doit s'exprimer de manière directe, sans détour. Souvent, ses textes ressemblent à des descriptions d'images et de situations anodines, de tous les jours; ils se terminent par une idée plus abstraite:

"Photo dans un journal"

Après la chute du shah

devant la prison centrale de Téhéran

des milliers de gens attendent les membres de leur famille.

Un père porte son fils sur ses épaules,

le fils tient une pancarte:

LIBERTÉ

Un homme attend sa femme.

Un fils

attend sa mère.

Et la liberté

qu'attend la liberté?

(Où je trépasse est mon ailleurs, p. 58)

Tant que son sujet est l'Iran, il parle de manière concrète. Mais dès qu'il se réfère à sa situation dans l'Ici et le Maintenant, c'est-à-dire à sa diaspora des trente dernières années, il recourt davantage à l'abstraction, comme s'il ressentait sa vie réelle à la manière d'un rêve, utilisant la métaphore:

Comme un poisson rouge

dans un verre translucide

je joue à la Méditerranée

et cultive mon culte du brochet.

De temps en temps seulement

j'embrasse la surface de l'eau

et je prétends

être en vie.

(Alors je crie jusqu'au silence, p. 14)

Le sentiment d'être marginalisé l'accompagne partout dans la diaspora. Mais quand, après la chute du shah, il retourne en Iran afin de participer à l'établissement d'un nouvel ordre politique, il se rend compte qu'il est ignoré, par son ancienne patrie d'abord, ensuite par la terre d'accueil. En Allemagne, il se brouille avec les 
co-révolutionnaires d'origine iranienne puisqu'il ne se laisse pas enfermer dans une étroite pensée politique. Ils le considèrent comme un traître, un renégat, et l'isolent à l'intérieur de leur communauté d'exil, ne l'écoutent ni lui parlent plus. Lentement, il doit accepter la durée indéfinie de son exil. Sans perdre son identité, il ne veut pourtant pas fuir la réalité. À la recherche de vérités nouvelles, il entreprend d'immenses lectures, et lit "plus que [son] cour ne supporte". SAID intègre dans sa pensée les sentiments des autres, mais il ressent le désir de formuler les siennes et de les communiquer. Mais pour qui? Pour ses camarades de la diaspora qui l'ont oublié et pour lesquels il n'éprouve guère de respect?

\author{
Seulement, \\ dans notre exil aride \\ personne ne voulait de mes poèmes persans. \\ Ils n'étaient que des chansons de bataille \\ annoncées dans notre cercle - \\ les exilés \\ ces eunuques doublement châtrés \\ qui tentent \\ de se masturber hérö̈quement en groupe.
}

(Autoportrait pour une mère lointaine, p. 20)

\title{
Écrire l'ailleurs, pour qui ?
}

Doit-il écrire pour ses "concitoyens allemands" qui l'ont pourtant ignoré jusqu'à présent? Il commence par s'adresser des lettres - mais en réalité, il cherche le dialogue et attend en vain des réponses. Finalement, il brise son isolement en se réfugiant dans la langue allemande. Il en fait la langue de ses poèmes, cette langue étrangère avec laquelle il a vécu pendant plus de la moitié de son existence, qui l'a accompagné de l'adolescence à la maturité. Il doit s'approprier cette langue, apprendre à la manier. En lançant ses messages littéraires à la mer, il attend des réponses: dans ses livres, il donne toujours son adresse de poste restante. Le numéro 4310 18, D - 80740 Munich acquiert une importance presque mystique pour lui puisqu'il le relie au monde extérieur. Il le garde même pendant son dernier voyage en Iran. La visite à un bureau de poste à Munich devient un rituel autour duquel s'organisent les événements de sa vie. Il y reçoit le courrier de ses lecteurs qui lui racontent des faits de 
116

leur vie privée, souvent bouleversants. Il cite la lettre d'une Belge après que son ami, un réfugié politique, s'est enlevé la vie. Avec l'aide de SAID, elle espère pouvoir écrire une lettre à la mère de celui dont le corps a été transféré en Iran (Le bras des mullabs, p. 128 et suiv.).

Les traces de la langue persane se retrouvent continuellement dans l'allemand de SAID, intégrées tout naturellement. Dans le poème "Maintenant tu as été exécuté", il utilise lors d'une conversation téléphonique avec le frère de Mehrdad Farjad, torturé, puis exécuté, les termes "Que ceci soit ton dernier deuil" (Le bras long des mullahs, p. 68 suiv.). Utiliser, comme cela se ferait en allemand ou en français, l'expression "mes condoléances", détruirait le caractère particulier du message. (Par ailleurs, le poète s'approprie volontiers des expressions calquées sur la langue allemande, comme par exemple "das Blaue vom Himmel herunterlügen", en français "mentir comme un arracheur de dents".)

\section{Relations avec les littératures nationales}

Une partie du recueil Où je trépasse est mon ailleurs a été publiée en 1987. Cependant, ces poèmes renvoient à des événements survenus en 1979. Il s'agit de la rétrospective d'un voyage en Iran (postrévolutionnaire), que SAID a entrepris "après 5111 nuits dans l'exil" avec la poétesse allemande Luise Rinser. Ces textes, fragmentés, sont adressés à une bien-aimée, restée en Allemagne, mais qui l'accompagne en pensées:

Ma bien-aimée,

dans ces rues je ne puis même pas tenir ta main.

Comme on se moque ici

de l'amour.

Où je trépasse

est mon ailleurs.

(Oü je trépasse est mon ailleurs, p. 62)

Ce voyage dans son ancienne patrie commence par la joie de retrouver son pays et avec un sentiment d'intimité. Il constate d'abord les possibilités d'un renouveau social et politique; mais bientôt les signes annonciateurs d'un nouveau culte de la personne et d'une dictature imminente le rendent morose et le déçoivent puisque le mouvement révolutionnaire est récupéré par 
les islamistes qui bannissent la gauche afin de la poursuivre à nouveau.

Dans Le bras long des mullabs, SAID présente de brefs portraits de personnes dont la vie a été profondément marquée par les bouleversements politiques dans l'histoire récente de l'Iran. La manière abrupte et factuelle de ces portraits indique un aspect particulier de la vie en exil, intérieur ou extérieur. Un exilé déraciné qui n'est pas intégré dans le pays d'accueil ressent le passage du temps comme très lent. Les jours et les semaines, vides, s'écoulent à pas de tortue. En rétrospective, ce même exilé ne comprend pas comment des périodes de longue durée ont pu lui glisser si rapidement entre les doigts. Ici, il s'agit d'un mécanisme psychologique qui fait partie du syndrome de l'exil : si une période de temps est remplie d'activités créatives, elle semble passer rapidement tout en gardant le caractère d'un écoulement rapide. Par contre, un temps mort et gaspillé donne une impression de lenteur qui s'inscrit dans la mémoire. C'est justement ce sentiment ambivalent du point de vue de l'exilé qui se traduit dans les brefs portraits brossés par le poète. Ainsi, le portrait de Tafreshian (dans Le bras long des mullabs, p. 98 et suiv.) permet de ressentir, avec le personnage, comme interminables l'emprisonnement et les longues années d'exil. Cependant, SAID résume cette éternité en quelques phrases, sans rien omettre. Le poète, restant lui-même à l'extérieur de l'histoire, peut cependant s'approprier la perspective intérieure de ces vies et nous la transmettre.

La lecture intensive de textes de la littérature mondiale est omniprésente dans l'cuvre de SAID. Le poète ne suit pas des modèles, comme l'avait fait Brecht, très prisé par ailleurs des intellectuels de gauche iraniens, mais il garde son caractère original. Toutefois, des parallèles thématiques entre les textes de SAID et ceux de ses auteurs préférés se retracent assez facilement. Ainsi, il parle souvent du passeport et de son importance pour l'identité de l'émigré:

Mais le passeport est devenu
dans l'ailleurs
notre troisième jambe
notre jambe de soutien.

(Autoportrait pour une mère lointaine, p. 50)

I1 est évident qu'il se rapproche ici de la première scène des Entretiens de réfugiés (Flüchtlingsgespräche) de Bertolt Brecht, 
118

même si l'attitude personnelle et le style narratif de SAID se distinguent de la manière distancée et ironique de Brecht. ( $C f$.: Flücbtlingsgespräcbe I. Über Pässe / Über die Ebenbürtigkeit von Bier und Zigarre / Über die Ordnungsliebe. Entretiens de réfugiés I. À propos des passeports / $\bar{A}$ propos de l'équivalence de la bière et du cigare / $A$ propos du goût de l'ordre ${ }^{1}$. Ailleurs, il renvoie de façon directe ou indirecte à des textes de poètes modernes iraniens:

Quand on arrêta Vartan et qu'il garda le silence,

les émissaires du pouvoir lui posèrent

une foreuse sur la tête

afin de trouver la vérité -

Vartan ne dit rien.

(Où je trépasse est mon ailleurs, p. 54)

SAID relate ici une histoire vraie, celle de l'arrestation et de la torture du dirigeant communiste Vartan après le coup d'État militaire de 1953 contre le gouvernement de Mohammad Mossadeq. Ahmad Schamlu, célèbre poète iranien moderne, avait écrit dans un hymne célébrant la mémoire de Vartan, la phrase "Vartan ne dit rien ". Le poème de Schamlu, qui appartient à la catégorie de la poésie des martyrs, est un exemple des textes que la gauche iranienne a empruntés à la tradition shi'ite. Bien que SAID soit moins enclin que d'autres poètes à suivre ce culte moderne des morts, il parle de héros ayant sacrifié leur vie pour une cause, sans oublier toutefois les contradictions de leur personnalité. Pour lui, il ne s'agit pas de monuments sans faille mais d'êtres humains en chair et en os qui ont donné leur sang, même si leur idéal était erroné ou si leurs dirigeants les ont trahis. À ce sujet, rappelons à nouveau le texte "Maintenant tu as été exécuté" dans lequel SAID entreprend un dialogue avec un fonctionnaire communiste qu'il connaissait du temps d'avant la révolution. Il y retrace l'histoire du machiavélisme des communistes iraniens qui voulaient s'unir aux islamistes afin de forger un "consensus antiimpérialiste et anticapitaliste" pour trouver la mort dans un bain de sang.

1. Bertolt Brecht, Werke 14, Prosa 4, Suhrkamp, Frankfurt s.Main, 1990, p. 1383 et suiv. 


\section{L'amour}

Pour SAID, l'amour est le contrepoids à son déracinement et à la perte de sa patrie. Il y cherche protection et repos:

Ton sourire soleil qui réchauffe la diaspora [...]

Nous sommes tous des Juifs éternels.

J'ai la nostalgie de tes bras.

(Poèmes d'amour; sans pagination)

Mais il sait que son destin est de rester toujours à l'extérieur et de ne pas pouvoir surmonter une distance infranchissable, comme lorsqu'il dit:

Je joue du piano avec des gants, j'écris mes poèmes dans des langues étrangères et j'embrasse ma bien-aimée à travers un verre opalin.

(ibid.)

Ces poèmes sont plus ancrés dans la tradition européenne que dans la persane. Mais à nouveau il ne peut être fixé dans une littérature "nationale" ou une tendance en particulier. Des sujets comme l'émancipation des femmes, l'opposition des sexes ou encore des références à des biens matériels, souvent au centre de la poésie allemande contemporaine, sont complètement absents de la poésie de SAID. D'un autre côté, la prédisposition du moi lyrique à traiter des idées et des sentiments personnels de manière ouverte et immédiatement accessible pour le lecteur est sans précédent dans la poésie iranienne d'aujourd'hui. Il est tout à fait possible que le concept amoureux selon le poète contemporain Farrokhzad, qui se laisse assez facilement déduire des formes de l'amour au quotidien, soit semblable à celui de SAID. Mais il serait insensé de vouloir établir Farrokhzad comme source d'inspiration: vu la variété et l'étendue des lectures de SAID, il n'est pratiquement jamais possible de réduire sa poésie à des sources ou des modèles précis. S'il était traduit en persan, 
120

ses poèmes d'amour détonneraient dans la poésie nationale actuelle.

Ici encore SAID démontre son caractère unique, issu de son existence, de sa philosophie cosmopolite et de différentes cultures.

Traduit de l'allemand par Hans-Jürgen Greif 